\title{
Data-driven Chaos Indicator for Nonlinear Dynamics and Applications on Storage Ring Lattice Design
}

\author{
Yongjun $\mathrm{Li}^{\mathrm{a}, *}$, Jinyu Wan ${ }^{\mathrm{b}, \mathrm{c}}$, Allen Liu ${ }^{\mathrm{d}}$, Yi Jiao ${ }^{\mathrm{b}, \mathrm{c}}$, Robert Rainer ${ }^{\mathrm{a}}$ \\ ${ }^{a}$ Brookhaven National Laboratory, Upton, New York 11973, USA \\ ${ }^{b}$ Institute of High Energy Physics, Beijing 100049, China \\ ${ }^{c}$ University of Chinese Academy of Sciences, Beijing 100049, China \\ ${ }^{d}$ Department of Electrical and Computer Engineering, Purdue University, West Lafayette, \\ Indiana 47907, USA
}

\begin{abstract}
A data-driven chaos indicator concept is introduced to characterize the degree of chaos for nonlinear dynamical systems. The indicator is represented by the prediction accuracy of surrogate models established purely from data. It provides a metric for the predictability of nonlinear motions in a given system. When using the indicator to implement a tune-scan for a quadratic Hénon map, the main resonances and their asymmetric stop-band widths can be identified. When applied to particle transportation in a storage ring, as particle motion becomes more chaotic, its surrogate model prediction accuracy decreases correspondingly. Therefore, the prediction accuracy, acting as a chaos indicator, can be used directly as the objective for nonlinear beam dynamics optimization. This method provides a different perspective on nonlinear beam dynamics and an efficient method for nonlinear lattice optimization. Applications in dynamic aperture optimization are demonstrated as real world examples.

Keywords: data-driven chaos indicator, surrogate model, nonlinear dynamics, dynamic aperture
\end{abstract}

\footnotetext{
* Corresponding author

Email address: yli@bnl.gov (Yongjun Li)
} 


\section{Introduction}

It is well-known that the predictability of motion in a nonlinear dynamical system is closely associated with its degree of chaos. Given an initial condition, although its motion is deterministic, its long-term prediction might not be quantitatively accurate because numerical errors can be cumulative and amplified. The Lyapunov exponent [1, i.e., the exponential growth of separation of infinitesimally close trajectories, is often used as a chaos indicator to characterize the sensitivity of chaotic motion to its initial condition.

Consider a different scenario: an unknown nonlinear dynamical system is encapsulated into a blackbox and only an ensemble of trajectories (input and output data) are available. Comparing actual trajectories to interpolated trajectories is one way to gauge chaos. A typical method to interpolate from known trajectories is to build a surrogate model with machine learning techniques. A surrogate model needs to be established first, then predictions can be made by evaluating trajectories with given initial conditions. This procedure is known as "supervised learning" 2]. To validate the model, the data is often randomly split into two clusters: a large training set and a small testing set. A model is then constructed from the training set. The performance of the model, i.e., the prediction accuracy, is measured by comparing the testing data against its prediction. The performance of the model depends on the type and complexity of the model, the volume of training data, the algorithm used for training, etc. Nevertheless, the prediction accuracy depends greatly on the degree of chaos. Therefore, an intuitive method for detecting chaos directly, purely from data is possible. In other words, predictability itself can act as a chaos indicator. From our studies we observed that by using the predictability of less-complex surrogate models, and a small volume of training data, some nonlinear behaviors in a dynamical system can be well characterized.

Surrogate models have been widely used in studying nonlinear dynamical systems [3, 4, 5, 6, 7, 8, including charged particle motion in modern accelerators 9 , 10, 11, 12, 13, 14, 15. These models are obtained by training on either 
simulated data or experimental data, which have a high computational demand or require complicated experimental processing. If models can predict the dynamical system properties accurately with reduced resource requirements, they can be used for more efficient applications, such as optimization problems. Improving the prediction accuracy is the highest priority in these applications. In contrast to these existing approaches, the main advantage of using data-driven chaos indicators is that the requirement on the absolute accuracy of surrogate models is less demanding, and therefore can be structured with less complexity and data.

To further explain this approach, the remaining sections are outlined as follows: in Sect.2 the detailed data-driven chaos indicator concept is introduced and applied to implement a tune-scan for a quadratic Hénon map. Sect. 3 correlates the nonlinear dynamical behavior of charged particles circulating in a storage ring with the data-driven chaos indicator, constructed with an artificial neural network (ANN) [16. In Sect. 4, we demonstrate the application of chaos indicators to optimize storage ring dynamic apertures. The model selection problem and other related issues are discussed in Sect. 5 A brief summary is given in Sect. 6 .

\section{Data-driven chaos indicator for Hénon map}

The well-studied quadratic Hénon map, as shown in Eq. (11), is used as an example to demonstrate how to construct a data-driven chaos indicator for tunescanning. It represents a thin sextupole kick followed by a linear rotation in a 2-dimensional phase space,

$$
\left(\begin{array}{l}
x \\
p
\end{array}\right)_{n+1}=\left(\begin{array}{cc}
\cos 2 \pi \nu & \sin 2 \pi \nu \\
-\sin 2 \pi \nu & \cos 2 \pi \nu
\end{array}\right)\left(\begin{array}{c}
x \\
p-\lambda x^{2}
\end{array}\right)_{n},
$$

where, $n$ is a non-negative integer, $\nu$ is known as the linear tune of the transformation, and the sextupole strength $\lambda$ is set as one for this demonstration. We assume the map in encapsulated as a blackbox with its tune as the control knob. For a given tune, as illustrated in Fig. 1, some known Trajectories 
that start with initial conditions $\left(x_{0}, p_{0}\right)$ (input data) within a specific area $x_{0}, p_{0} \in[-0.6,+0.6]$, end with $\left(x_{n}, p_{n}\right)$ (output data) after a limited number of turns (such as $n=20$ ). Based on the data, we can extract some parameters to characterize its long-term stability such as, the location of resonance lines and their stop-band widths, the relative size of the stable region, etc. This is accomplished by carrying out a tune-scan.

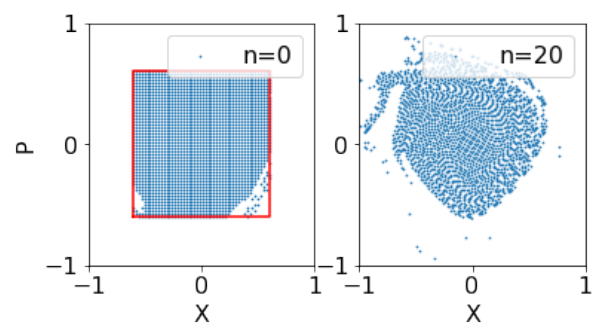

Figure 1: A short-term (20 turns) coordinate transformation of the Hénon map with $\nu=$ 0.205. The initial conditions (left) are populated within the red square $(x, p) \in[-0.6,+0.6]$. The blank points represent unstable trajectories, where at least one of their $x$ and $p$ amplitudes exceed a threshold 10 during the transformation. The transformed coordinates that survived after 20 turns are shown in the right subplot.

A tune-scan can be used to compare a nonlinear system's behavior at different linear tunes. At each given tune, some trajectories are produced from the blackbox. Most of them (around 80-90\%) are used to train a surrogate model with a polynomial regression algorithm,

$$
\left(\begin{array}{c}
x \\
p
\end{array}\right)_{n}=\left(\begin{array}{lllll}
a_{0} & a_{1} & a_{2} & a_{3} & a_{4} \ldots \\
b_{0} & b_{1} & b_{2} & b_{3} & b_{4} \ldots
\end{array}\right)\left(\begin{array}{c}
1 \\
x \\
p \\
x^{2} \\
x p \\
p^{2} \\
\vdots
\end{array}\right)_{0}
$$

Here, the $7^{\text {th }}$ order polynomials are used. The rest, $10-20 \%$ of the data, is used as a testing set for performance validation. The validation is done by comparing 
the testing data against their model predictions (Fig. 2). Quantitatively, the prediction accuracy is measured with the mean squared errors (MSE) between the predictions and the true values. It also serves as the data-driven chaos indicator.
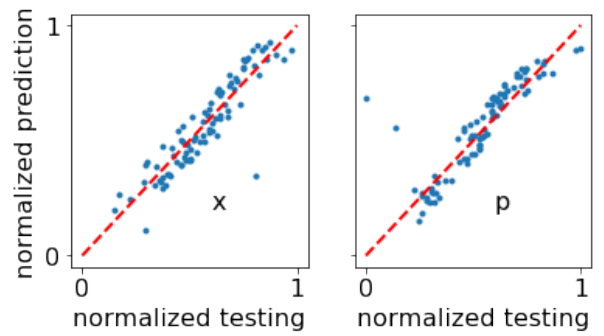

Figure 2: Prediction performance of $7^{\text {th }}$-order polynomials surrogate model for the Hénon map in the $x$ and $p$ plane at a given tune $\nu=0.205$. Each dot represents one initial condition in the testing cluster. The red dashed line is the desired/expected value. Note the original data has been scaled/normalized to a range of $[0,1]$.

The tune-scan result is illustrated in Fig. 3 in which the model's prediction performance is shown as the blue line with error bars. For each tune, the data was re-sampled randomly into different training/testing sets, multiple times (also known as the cross-validation technique). The shuffling of data can avoid selecting data that is trapped in a specific resonance, preventing the degree of chaos from being under- or over-estimated. The error bars represent the statistical fluctuations with different re-samplings. Due to quadratic perturbation, the worst model prediction occurs at $\nu=\frac{1}{3}$ as expected, which corresponds to a strong $3^{r d}$-order resonance line. This resonance also has the widest stop-band width (approximated by the width of half-height of peak). Besides $\frac{1}{3}$, some other high order resonances at $\nu=\frac{1}{4}, \frac{1}{5}$, even $\frac{1}{7}$ are visible with this chaos indicator. For comparison, a long-term (2,048 turns) transformation starting from a wide initial condition of the $x$ and $p$ within $[-1.2,+1.2]$ was computed. Its loss (i.e., unstable trajectory) rate as the function of the tune is also shown as the red solid line in Fig. 3. The data-driven chaos indicator observed appears to be highly correlated with the loss rate of the long-term tracking. 


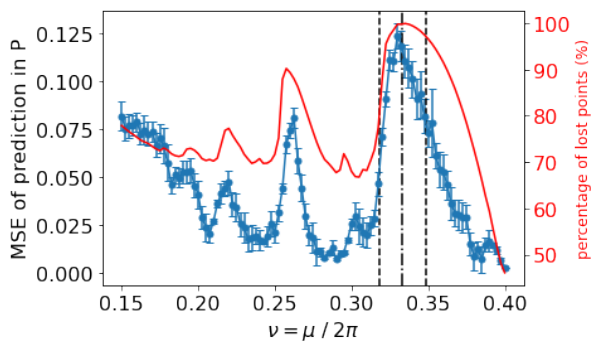

Figure 3: Prediction performance of a polynomial surrogate model (blue line with error bars) vs. loss rate (solid red) of Hénon map at different tunes. The black dot-dash line denotes the location of the $\frac{1}{3}$ resonance line and two dashed lines mark out its asymmetric stopband widths at each side with a tune separation of \pm 0.015 .

It is interesting to note that an asymmetric stop-band width is detectable with this chaos indicator in Fig. 3. The appearance of the asymmetry is due to amplitude dependent detuning. It behaves differently when the linear tune is slightly off the resonance line as shown in Fig. 4. When the linear tune is below the $\left(\frac{1}{3}\right)^{-}$resonance (in the left side), its amplitude-dependent tunes drift away from the resonance (dashed line). Therefore, the motions are less chaotic, and the left stopband width is narrow. But when the linear tune is above the $\left(\frac{1}{3}\right)^{+}$(in the right side), its amplitude-dependent tune merges to the resonance quickly (solid line), so the motions are more chaotic, and the stopband width at the right side is correspondingly wide. This asymmetry is also observed at $\nu=\frac{1}{4}, \frac{1}{5}$ and confirmed with the loss rate.

The two tune-scan results in Fig. 3 are closely correlated. The information needed for these, however, can be very different. Using the data-driven chaos indicator, even short-term (20 turns) map transformations for only partial initial conditions can provide some useful information. The tune-scan using the loss rate is more accurate, but it also requires a greater number (2,048 turns) of map transformations for more initial conditions of $x$ and $p$. In real-world applications, there may be a high resource demand to obtain such data. Using limited data resources to obtain an early chaos indicator has the potential advantage of boosting the optimization of design of a nonlinear dynamical system. 


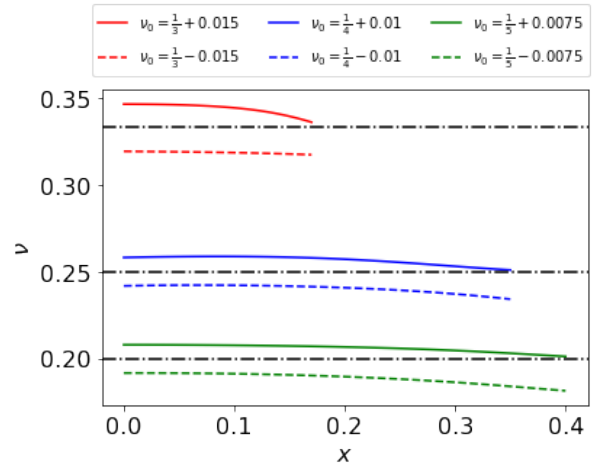

Figure 4: Different behaviors of the amplitude-dependent detuning when the linear tune approaches the $\frac{1}{3}, \frac{1}{4}, \frac{1}{5}$ resonances, which results in asymmetric stop-band widths. The solid lines indicate when the linear tune is slightly above a resonance, and the amplitude-dependent tunes merge to the resonance line (the dot-dash lines) quickly. The dashed lines are for the linear tune below the resonances, in which amplitude-dependent tune drifts away from the resonance. This effect leads to asymmetric and shifted peaks around the resonances. The asymmetric detuning effect can be detected by both the data-driven chaos indicator and a detailed loss rate tracking in Fig. 3 .

\section{Data-driven chaos indicator for storage rings}

When studying the stability of charged particles in modern storage rings, implementation of particle tracking is often necessary. To determine the dynamic aperture (DA) boundary, in which particle motion is stable would require this type of simulation. During the design stage, many time-consuming tracking simulations are needed to search for magnet lattices with sufficient DA. To boost the search process, surrogate models have been used to replace the original accelerator lattices [10, 12, 13, 14]. The accuracy of the models usually plays a critical role in ensuring optimization converges in the desired direction. Modern accelerators are integrated with some strong nonlinear magnets. This can make reliable surrogate models difficult to construct. From another perspective, as mentioned previously, the prediction accuracy of surrogate models can be a metric for the chaos of particle motion.

Consider a storage ring accelerator composed of various magnetic elements, 
in which the transportation of a charged particle for single turn (or a few repetitive turns) can be represented by a nonlinear transformation

$$
\vec{X}_{1}=M_{0 \rightarrow 1} \cdot \vec{X}_{0}
$$

Here, $\vec{X}_{1}, \vec{X}_{0}$ are the particle coordinates in the phase space, and $M_{0 \rightarrow 1}$ is the one-turn transportation map. Given the ring magnetic lattice, and using some simulated trajectory data $\left(\vec{X}_{0}, \vec{X}_{1}\right)$ as illustrated in Fig. 5 , a surrogate model constructed with an artificial neural network (ANN) as shown in Fig. 6 has been adopted. The reason for using a different model here is to indicate that a datadriven chaos indicator represents the predictability of the system regardless of the selection of models. For this investigation, a simulated trajectory dataset was divided into a training set and a testing set. The accuracy of the model was still measured by the mean squared error (MSE) between the testing set and its model prediction.
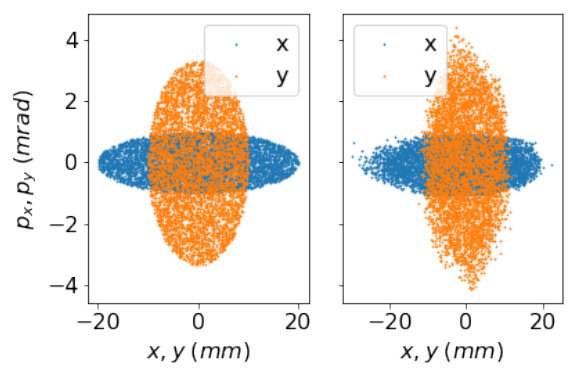

Figure 5: Simulated one-turn transportation of a storage ring lattice with some given inputs (left) and their outputs (right) in the 4-dimensional phase space. The ranges of inputs are comparable to the desired dynamic aperture there. This is the input and output data that the ANN is trained on.

Using the National Synchrotron Light Source II (NSLS-II) storage ring [17] as an example, four special scenarios were studied to demonstrate the proof of concept, illustrated in Fig. 7. First, consider a special scenario in which the lattice does not include any nonlinear magnets. The one-turn map is linear. In this case the model can accurately predict any testing trajectory. As the system 


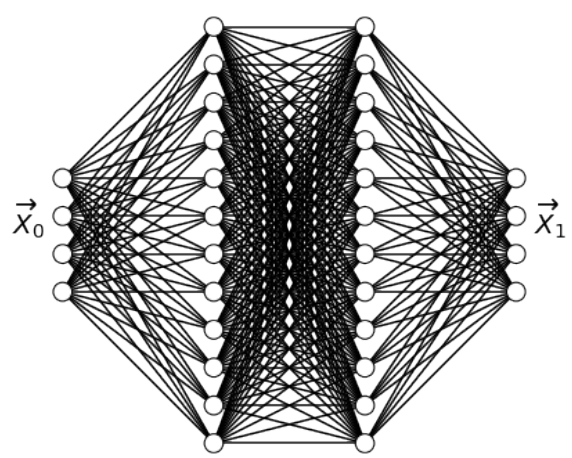

Figure 6: A four-layer $4 \times 12 \times 12 \times 4$ ANN used as the surrogate model to represent a storage ring's one-turn transportation in the 4-dimensional phase space.

is a simple linear transformation, the level of accuracy is only limited by the computational numerical error, as shown in the first row of Fig. 7 .

A linear lattice, however, cannot maintain stable beam at a high current due to its intrinsic negative chromaticity [18]. The chromaticity represents a particle oscillation frequency shift due to its energy deviation. Therefore, some nonlinear sextupole magnets must be integrated into the lattice to compensate for the inherent chromaticity. In the second scenario of our example, the chromaticity was corrected to a value of positive 2 using chromatic sextupoles. After the correction, the same structured ANN model saw significantly reduced prediction accuracy. This was because particle motions became chaotic, and even unstable, when their trajectory diverged far from the origin. Due to strong nonlinearity, many particles fail to survive even for a single turn under these conditions. In the second scenario of Fig. 7, low density clusters with large prediction discrepancies were observed. Only those survived test particles are plotted out.

In the third scenario of Fig. 7, some harmonic sextupoles were included to compensate for the nonlinearity caused by the chromatic sextupoles. These sextupoles were not fully optimized, therefore, limited improvements to the accuracy were seen. In the last scenario, after the harmonic sextupoles were 

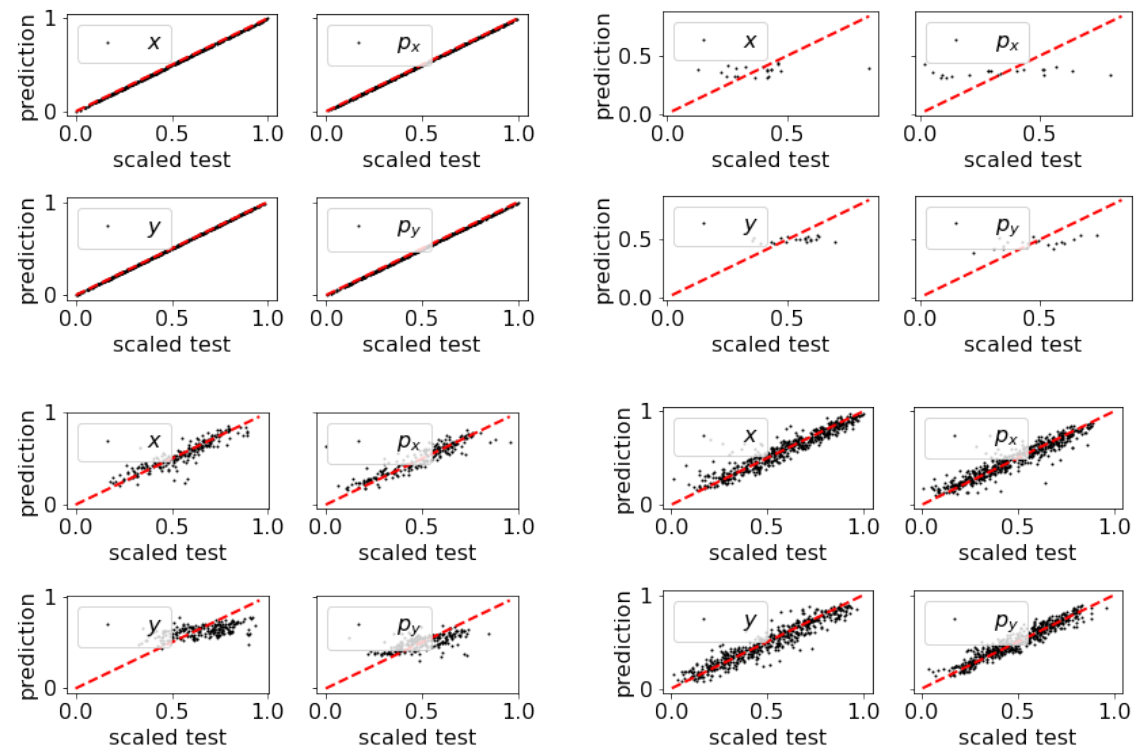

Figure 7: Prediction accuracies of the ANN model in four different scenarios with different sextupole settings. Each subplot in every row (scenario) represents one of the projected dimensions $\left(x, p_{x}, y, p_{y}\right)$, in which a black dot represents one particle's scaled coordinate. The dashed red line represents the desired value. Top left: a perfect surrogate model for a purely linear lattice (black dots and the dashed red lines overlap); Top right: a lattice with only chromatic sextupoles in place, the prediction accuracies are poor; Bottom left: improved accuracies are seen after introducing some harmonic sextupoles, but the settings are not optimal; Bottom right: a lattice with optimized harmonic sextupoles, the prediction accuracy is greatly improved.

optimized by minimizing the data-driven chaos indicator (as will be explained later), the chaos was significantly reduced and most simulated particles survived for at least a single turn. Consequently, the prediction accuracy of the ANN model was greatly improved in the last scenario, in which high density clusters were distributed around the desired prediction line.

The correlations observed in the previous example indicate that the prediction accuracy of a surrogate model can be used as a chaos indicator for a complicated storage ring system. For a given surrogate model and a certain amount of training data, the model prediction becomes more inaccurate when 
particle motion in a lattice is more chaotic. Therefore, rather than constructing complex, high performance models, the prediction accuracy itself can be used as the objective for nonlinear lattice optimization. Another advantage is that the computational resources for the data analysis (for single turn trajectories) are greatly reduced, compared with an accurate DA computation through multi-turn (often thousands of turns) tracking.

\section{Applications in dynamic aperture optimizations}

In this section the prediction accuracy of ANN models, used as a chaos indicator, was applied to optimize two different types of nonlinear lattices: the existing NSLS-II ring with double-bend achromat (DBA) lattice and a complex ESRF-EBS [19] type hybrid multi-bend achromat (MBA) lattice.

\subsection{NSLS-II's DBA Lattice}

The NSLS-II [17] is a dedicated $3^{\text {rd }}$ generation medium energy $(3 \mathrm{GeV})$ light source operated by Brookhaven National Laboratory. Its main storage ring lattice is a traditional DBA structure as illustrated in Fig. 8. After the linear chromaticity is corrected by chromatic sextupoles, the available "tuning knobs" used for DA optimization are six families of harmonic sextupoles.

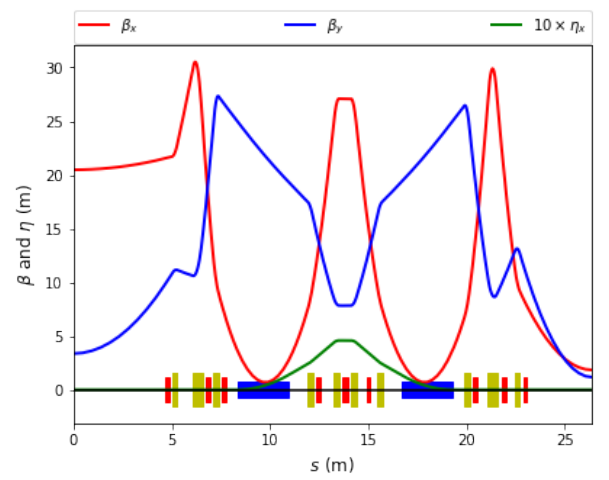

Figure 8: Linear optics and magnet layout for one DBA cell of the NSLS-II storage ring. Red blocks represent sextupoles, blue ones are dipoles, and yellow ones are quadrupoles. 
A four-layer $4 \times 12 \times 12 \times 4$ ANN was chosen as the surrogate model to represent a one-turn transportation for on-momentum particles. The desired DA dimensions are $x=25 \mathrm{~mm}$ and $y=10 \mathrm{~mm}$ in the horizontal and vertical planes respectively, at the location of injection point. Thus, two elliptical areas in the phase space with axes at $\left(x, \frac{x}{\beta_{x}}\right)$ and $\left(y, \frac{y}{\beta_{y}}\right)$, are uniformly populated with 5,000 initial conditions as the input, $\vec{X}_{0}$. Here $\beta_{x, y}$ are the local Twiss parameters [20]. The one-turn transportation can be accomplished with a symplectic particle tracking code, such as ELEGANT [21. The coordinates at the exit are the output $\vec{X}_{1}$. The volume ratio of the training and testing data is 90\%:10\%. The python packages SCIKIT-LEARN [22] and KERAS [23] have been used for this application. To avoid over- or under-fitting, the maximum number of training epochs was set to a sufficiently large number, and an early stopping point was used to halt the training once the model performance ceased improving. The mean squared error (MSE) in each phase space dimension is used as an independent optimization objective. By varying harmonic sextupole settings, the accuracy of the ANN model was monitored and used to drive a multi-objective genetic algorithm (MOGA) optimizer [24]. Besides using the four MSEs in each dimension $\left(x, p_{x}, y, p_{y}\right)$ as the objectives, a minimum number of confined trajectories in the ensemble is used as a constraint. In other words, for a given sextupoles configuration, only when at least $80 \%$ of trajectories are confined within a predefined range $x, y \in[-0.1,0.1] m$, it can be considered as a qualified candidate. A good convergence of the average prediction accuracy was reached after a $100^{\text {th }}$ generation of evolution as shown in Fig. 9. Using 100 Intel ${ }^{\circledR}$ Xeon ${ }^{\circledR}$ 2.2-2.3 GHz CPU cores, optimization on this scale takes about 6 to 8 hours.

The DAs of the total population in the $100^{\text {th }}$ generation were calculated. The histogram of their DA areas in Fig. 10 shows that most of candidates have a sufficient DA, with the smallest ones being greater than $400 \mathrm{~mm}^{2}$, which are more than sufficient to satisfy the requirements of machine operations. The existing operation lattice DA was also marked for a comparison.

To further confirm the correlation between the DA area and the ANN model's 


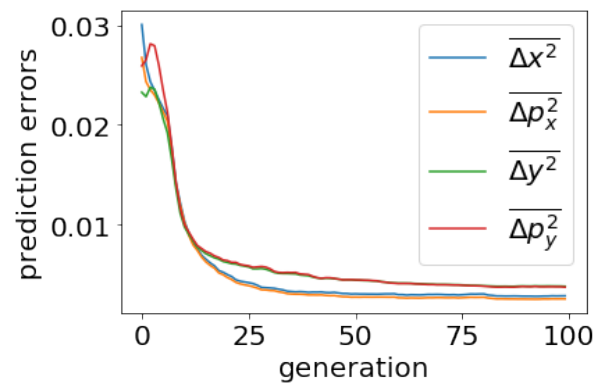

Figure 9: Convergence of prediction accuracy measured with four mean squared errors of test datasets in the surrogate/MSE MOGA optimization.

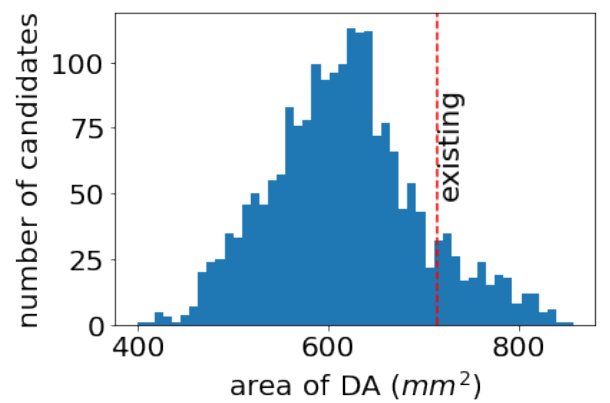

Figure 10: Distribution of the DA areas in the last generation of the surrogate/MSE enhanced-MOGA optimization. The location of dashed line marks the DA area for the existing operation lattice 17

accuracy, The DA areas and average prediction accuracy for all candidates in the $1^{\text {st }}$ and $100^{\text {th }}$ MOGA generations were computed for comparison. As shown in Fig. 11, the settings for the $1^{\text {st }}$ generation have relatively poor model prediction accuracy, and their DAs are small. Meanwhile, prediction accuracy in the $100^{\text {th }}$ generation is significantly improved, as well as their DAs. Using this chaos indicator, which requires limited computation time and resources, significantly boosted the nonlinear lattice optimization.

The candidate with the largest on-momentum DA was chosen to implement a detailed frequency map analysis (FMA) [25] as shown in Fig. 12. The DA comparison between this candidate and our existing operation lattice is shown in Fig. 13. An experimental test with live beam has been also carried out to 

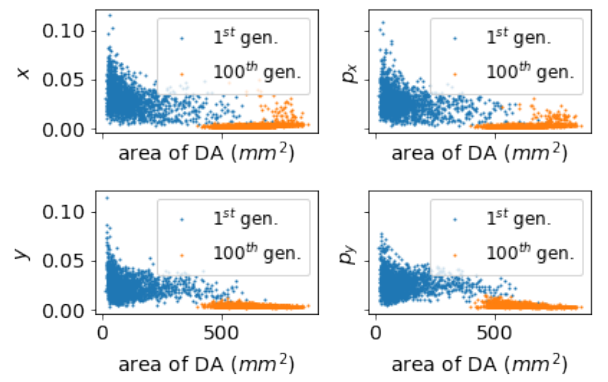

Figure 11: Correlation between the dynamic aperture and the ANN prediction accuracy seen in the MOGA optimization. The vertical axis in each subplot is the prediction accuracy in the corresponding dimension. The surrogate models of the $1^{\text {st }}$ generation (Blue dots) have low accurate predictions due to greater chaotic motion, and therefore, their original lattices have smaller DAs. Meanwhile, prediction accuracies of the $100^{\text {th }}$ generation are improved, as are their DAs.

confirm that this nonlinear lattice satisfies the requirements on the off-axis topoff injection and beam lifetime. Its DA is comparable to the solutions found using other methods [26, 27, 28, 29].

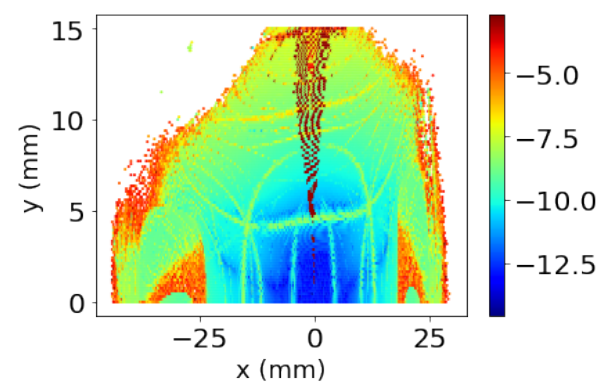

Figure 12: On-momentum dynamic aperture colored with the diffusion, obtained from frequency map analysis for the candidate with the largest DA. The color represents the tune diffusion $\log _{10}\left(\Delta \nu_{x}^{2}+\Delta \nu_{y}^{2}\right)[25$.

Each chaos indicator in the 4-dimensional phase space is used as an independent and equally important optimization objective. Therefore, it is not surprising to find that some optimized results have different prediction accuracies in the horizontal and vertical planes. In the example illustrated in Fig. 14 the 


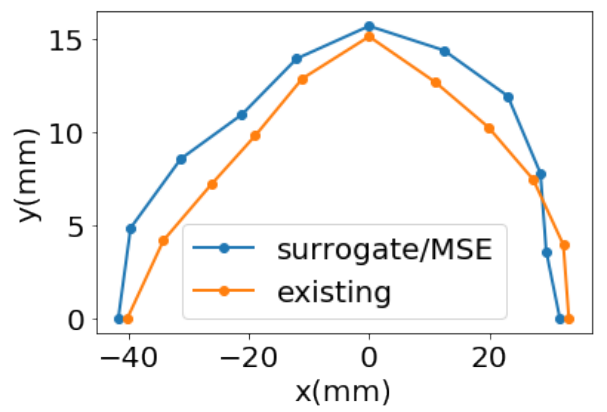

Figure 13: Comparison of on-momentum dynamic aperture between the best surrogate/MSE candidate (blue dots-line) and the existing operation lattice (yellow dots-line) [17.

vertical predictions are significantly worse than the horizontal ones. A detailed DA tracking simulation shows that the vertical amplitude-dependent detuning is larger than the horizontal one, which leads to more chaotic vertical motions within the DA. When the tune footprint crosses $6^{\text {th }}$ order resonances even at a relatively small vertical amplitude $y \approx 3 \sim 5 \mathrm{~mm}$, particle loss can occur. Although this result does not provide a good DA solution, it shows that a data-driven chaos indicator is capable of distinguishing resonance-driven chaos among different dimensions of phase space, even for complicated dynamical systems such as a modern storage ring.

Thus far, in the previous proof-of-principle study, only the on-momentum particles in the 4-dimensional transverse phase space are used for demonstration purposes. A sufficient off-momentum DA is of importance to ensure the beam lifetime. In an actual DA optimization, a 6-dimensional phase space should be considered. However, the longitudinal oscillation is relatively slow compared with the transverse ones. When designing a light source ring, usually a nonlinear lattice with fixed momentum-deviations are considered for DA optimization. In this case, the surrogate model's input have five components $\vec{X}_{0}=\left(x, p_{x}, y, p_{y}, \delta=\frac{\Delta p}{p_{0}}\right)$, but still four components in the output because $\delta$ is the same as its input. The settings for the MOGA optimization, such as the number of objectives and tuning knobs, are unchanged. Only the inputs of the 

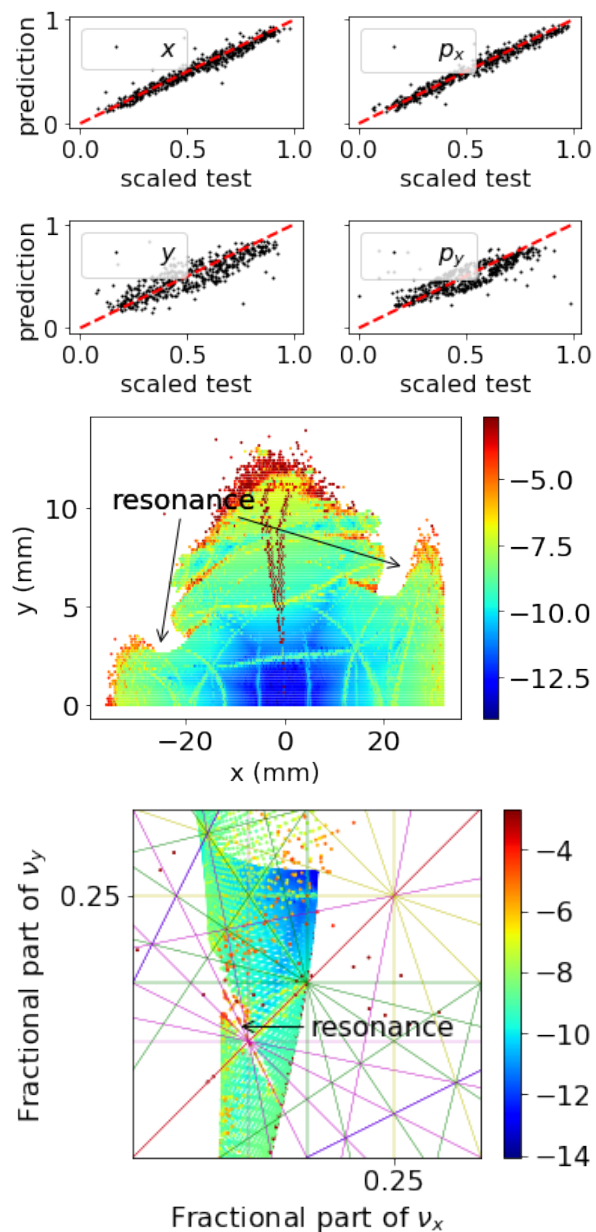

Figure 14: Top: good predictions in the horizontal plane, but poor ones in the vertical plane. Middle: DA crossing a resonance at small vertical amplitude can lead to particle loss due to more chaotic vertical motions. Bottom: Tune footprint in the tune space shows a large amplitude-dependent detuning in the vertical plane, which explains why the predictions in the vertical plane is worse.

ANN or polynominal's variables need to be expanded from four to five. The comparison of its on- and off-momentum DAs with the existing operation lattice is shown in Fig 15. The original and optimal sextupoles settings for the NSLS-II DBA lattice obtained with the 4- and 5-dimensional phase space are listed in Tab. 1. Even the six families harmonic sextupoles $(S H$ and $S L)$ settings are 
quite different, their DAs are still comparable. Since only single-turn tracking trajectories are used to train surrogate models to drive the MOGA optimizer, the computation cost of using the new technique is much lower.

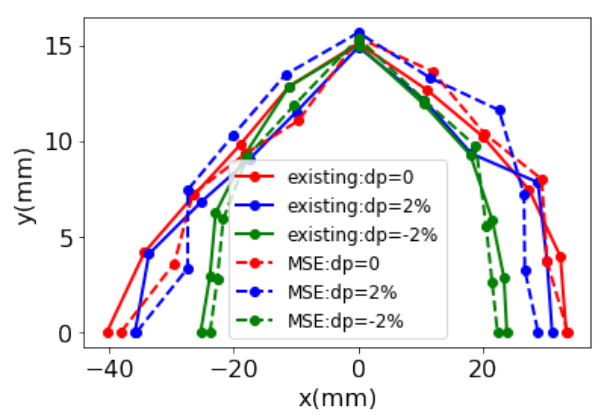

Figure 15: Comparison of on- and $\pm 2 \%$ off-momentum DAs between the existing NSLSII operation lattice (solid dot-lines) and the one optimized with surrogate/MSE in the 5dimensional phase space (dashed dot-lines). The sextupoles settings are listed in Tab. 1 .

Table 1: Sextupoles settings for existing lattice and obtained with 4- and 5-D surrogate/MSE

\begin{tabular}{|r|r|r|r|}
\hline Name & existing $K_{2}\left(m^{-3}\right)$ & $K_{2}\left(m^{-3}\right) 4 \mathrm{D}$ & $K_{2}\left(m^{-3}\right) 5 \mathrm{D}$ \\
\hline \hline SH1 & 19.83291 & 20.49257 & 23.73833 \\
\hline SH3 & -5.85511 & -1.14901 & -9.64248 \\
\hline SH4 & -15.82090 & -21.40682 & -14.99301 \\
\hline SL1 & -13.27161 & -2.85597 & -17.44383 \\
\hline SL2 & 35.67792 & 26.11824 & 34.05922 \\
\hline SL3 & -29.46086 & -24.72681 & -23.61317 \\
\hline
\end{tabular}

\subsection{Hybrid MBA Lattice}

Low-emittance light sources have entered a new era. Various multi-bend achromat (MBA) type lattices have been developed to reach diffraction-limited horizontal emittances. These lattices can deliver much brighter X-ray beams than seen before. In this section the data-driven chaos indicator was used to optimize the dynamic aperture of an MBA lattice type with seven bends, as shown in Fig. 16. 


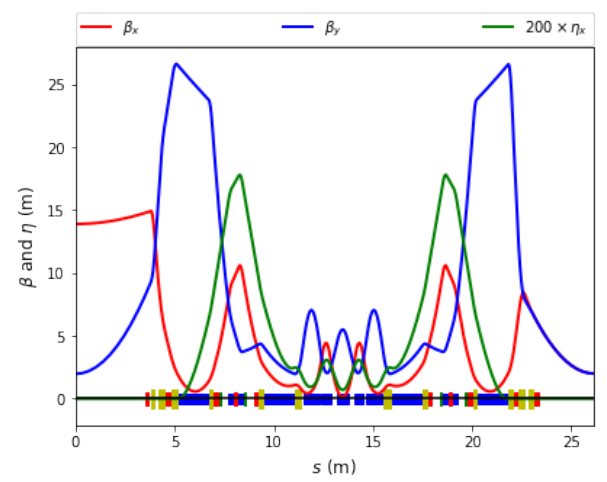

Figure 16: Linear optics and magnet layout for one cell of an ESRF-EBS type 19] MBA lattice. The lattice can provide a diffraction-limited horizontal emittance $30 \mathrm{pm}$ with a $3 \mathrm{GeV}$ beam. Red blocks are sextupoles, yellow ones are quadrupoles, and blue ones are either dipoles or off-axis quadrupoles (i.e., inverse bending magnets.)

In this example, several families of chromatic sextupoles were used. Maintaining constant linear chromaticities needs to be considered a constraint while tuning the sextupoles. Due to stronger sextupoles, the constructed surrogate model's prediction becomes worse than the previous DBA lattice. However, for an optimization problem, the chaos indicator only needs to provide relative values rather than absolute ones to guide the numerical optimizer to convergence. Similar convergence curves as Fig. 9 were observed but not shown here. One of the optimal DAs is shown in Fig. 17, which is sufficient for adopting an off-axis injection scheme at one of its long straight sections. This example shows that the data-driven chaos indicator can still be applied to a complex lattice design.

\section{Discussions on model selection and others}

Two different surrogate models were used in our studies. A $7^{\text {th }}$-order polynomial was used to study the Hénon map. In the examples provided by two storage ring lattices, a four-layer $4 \times 12 \times 12 \times 4 \mathrm{ANN}$ as shown in Fig. 6 was used. The selection of model complexity (the order in polynomial model, and the number of hidden layers and nodes in an ANN) compromise between model accuracy 


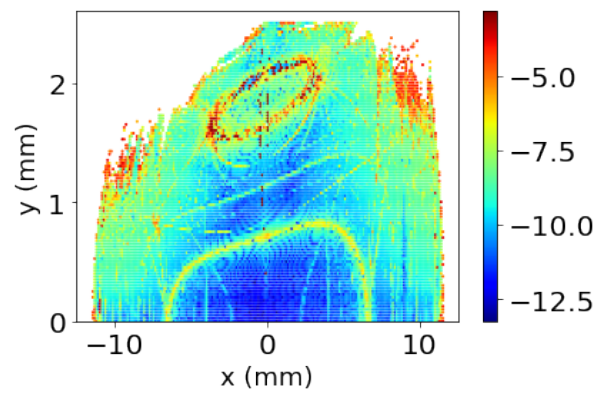

Figure 17: Dynamic aperture optimized with a data-driven chaos indicator for a complex MBA lattice. The color represents the tune diffusion.

and processing time. In principle, a more complicated model can achieve predictions with greater accuracy, but would need more data and time to train. For these applications, high absolute prediction accuracy is not critical because only a relative accuracy is needed to direct the optimizer to converge. For example, a three-layer ANN was also tested and confirmed functional for the lattice optimization. However, its statistical fluctuations were larger than the one used in the four-layer ANN and therefore were not the focus of the investigation. Although, at the early stages of optimization, a course, simple-structured model could be used to quickly narrow down the ranges of searching parameters. This would be much more computationally efficient. Then another, more complex model can be deployed for a finer, more accurate and precise search. Besides the application of an ANN and a polynomial regression, another surrogate model using the support vector regression [30] was also confirmed to be able to see the existence of correlations. Other surrogate models should be functional as well with this technique, although they were not tested in this investigation.

There are also some other advanced machine-learning techniques available that can better evaluate accuracy of an ANN model. For example, using the Kfold cross validation method [31] ensures that every observation from the original dataset has the chance of appearing in training and test sets, especially when limited input data is available. In our examples for lattice optimization, rather than using the time-consuming cross-validation that K-fold requires, sufficient 
training data were generated with a particle tracking simulation code, because using only one-turn tracking for such rings requires much less of a demand on computational resources.

The original Hénon map and particle transformation are symplectic, i.e., they represent conservative Hamiltonian systems, while the surrogate models established from data are usually not. The lack of symplecticity can result in artificial damping or excitation in long-term transformations. The established surrogate models should not be used for the DA computation. However, while constructing data-driven chaos indicators, surrogate models were only used to characterize the sensitivity of transformations to their initial conditions. Even if the transformations are not perfectly symplectic, it does not affect such applications.

Due to the lack of a real physics model, this chaos indicator cannot replace the diffusion rate obtained with the FMA, which measures the regularity of resonant motions of a nonlinear dynamical system. Using this indicator as the optimization objective might not be as competitive as the direct tracking-based optimization if not taking the computation cost into account. This method should be used as a complementary (rather than an alternative) tool for DA optimization. Because of its computation efficiency, a much larger population size can be used to get some potentially good solutions or narrow down the searching ranges quickly. Then some accurate but time-consuming trackingbased optimization can be further implemented.

\section{Summary}

A novel data-driven chaos indicator concept was introduced by correlating the degree of chaos of a dynamical system and its surrogate model's prediction accuracy. This indicator can be used to optimize the dynamic aperture of storage rings. Traditionally, the prediction accuracy of a model has been critically important for many machine-learning applications. With this method, however, the prediction accuracy is used as a relative indicator of the chaos of a dynamical 
system. Greater accuracy is therefore less important, and surrogate models which have a lower resource demand are sufficient for this purpose. This method also provides a new perspective on the characterization of chaos in nonlinear dynamical systems and an efficient method for dynamic aperture optimization.

\section{Acknowledgements}

We would like to thank Dr. Y. Hao (MSU) and the colleagues from NSLS-II for stimulating discussions. This research is supported by the U.S. Department

of Energy under Contract No. DE-SC0012704 (BNL), National Natural Science Foundation of China (No. 11922512) and Youth Innovation Promotion Association of Chinese Academy of Sciences (No. Y201904). A. Liu is supported by Virginia Pond Scholarship Summer Intern Program at BNL (2019).

\section{References}

[1] A. Vulpiani, F. Cecconi, M. Cencini, Chaos: from simple models to complex systems, Vol. 17, World Scientific, 2009.

[2] T. M. Mitchell, et al., Machine learning (1997).

[3] M. Barahona, C.-S. Poon, Detection of nonlinear dynamics in short, noisy time series, Nature 381 (6579) (1996) 215-217.

[4] T. Schreiber, A. Schmitz, Improved surrogate data for nonlinearity tests, Phys. Rev. Lett. 77 (1996) 635-638.

[5] I. Tokuda, T. Miyano, K. Aihara, Surrogate analysis for detecting nonlinear dynamics in normal vowels, The Journal of the Acoustical Society of America 110 (6) (2001) 3207-3217.

[6] A. P. Deshmukh, J. T. Allison, Design of nonlinear dynamic systems using surrogate models of derivative functions, in: ASME 2013 International Design Engineering Technical Conferences and Computers and Information in 
Engineering Conference, American Society of Mechanical Engineers Digital Collection, 2013.

[7] K. Lindhorst, M. Haupt, P. Horst, Efficient surrogate modelling of nonlinear aerodynamics in aerostructural coupling schemes, AIAA Journal 52 (9) (2014) 1952-1966.

[8] C.-D. Han, B. Glaz, M. Haile, Y.-C. Lai, Adaptable hamiltonian neural networks, arXiv preprint arXiv:2102.13235 (2021).

[9] A. Sanchez-Gonzalez, P. Micaelli, C. Olivier, T. Barillot, M. Ilchen, A. Lutman, A. Marinelli, T. Maxwell, A. Achner, M. Agåker, et al., Accurate prediction of x-ray pulse properties from a free-electron laser using machine learning, Nature communications 8 (1) (2017) 1-9.

[10] F. Wang, M. Song, A. Edelen, X. Huang, Machine learning for design optimization of storage ring nonlinear dynamics, arXiv preprint arXiv:1910.14220 (2019).

[11] X. Huang, M. Song, Z. Zhang, Multi-objective multi-generation gaussian process optimizer for design optimization, arXiv preprint arXiv:1907.00250 (2019).

[12] A. Edelen, N. Neveu, M. Frey, Y. Huber, C. Mayes, A. Adelmann, Machine learning for orders of magnitude speedup in multiobjective optimization of particle accelerator systems, Phys. Rev. Accel. Beams 23 (2020) 044601.

[13] J. Wan, P. Chu, Y. Jiao, Neural network-based multiobjective optimization algorithm for nonlinear beam dynamics, Phys. Rev. Accel. Beams 23 (2020) 081601.

[14] M. Kranjčević, B. Riemann, A. Adelmann, A. Streun, Multiobjective optimization of the dynamic aperture using surrogate models based on artificial neural networks, Phys. Rev. Accel. Beams 24 (2021) 014601. 
[15] J. Zhu, Y. Chen, F. Brinker, W. Decking, S. Tomin, H. Schlarb, Highfidelity prediction of megapixel longitudinal phase-space images of electron beams using encoder-decoder neural networks, Phys. Rev. Applied 16 (2021) 024005.

[16] M. H. Hassoun, et al., Fundamentals of artificial neural networks, MIT press, 1995.

[17] BNL, NSLS-II preliminary design report, https://www.bnl.gov/nsls2/ project/PDR/.

[18] S.-Y. Lee, Accelerator physics, World scientific publishing, 2018.

[19] L. Farvacque, N. Carmignani, J. Chavanne, A. Franchi, G. Le Bec, S. Liuzzo, B. Nash, T. Perron, P. Raimondi, et al., A low-emittance lattice for the ESRF, Proc. IPAC'13 (2013) 79-81.

[20] E. D. Courant, H. S. Snyder, Theory of the alternating-gradient synchrotron, Annals of physics 3 (1) (1958) 1-48.

[21] M. Borland, Elegant: A flexible sdds-compliant code for accelerator simulation, Tech. rep., Argonne National Lab., IL (US) (2000).

[22] F. Pedregosa, G. Varoquaux, A. Gramfort, V. Michel, B. Thirion, O. Grisel, M. Blondel, P. Prettenhofer, R. Weiss, V. Dubourg, J. Vanderplas, A. Passos, D. Cournapeau, M. Brucher, M. Perrot, E. Duchesnay, Scikit-learn: Machine learning in Python, Journal of Machine Learning Research 12 (2011) 2825-2830.

[23] F. Chollet, et al., Keras, https://keras.io (2015).

[24] K. Deb, A. Pratap, S. Agarwal, T. Meyarivan, A fast and elitist multiobjective genetic algorithm: Nsga-ii, IEEE transactions on evolutionary computation 6 (2) (2002) 182-197.

[25] J. Laskar, Introduction to frequency map analysis, in: Hamiltonian systems with three or more degrees of freedom, Springer, 1999, pp. 134-150. 
[26] L. Yang, Y. Li, W. Guo, S. Krinsky, Multiobjective optimization of dynamic aperture, Physical Review Special Topics-Accelerators and Beams 14 (5) (2011) 054001.

[27] Y. Li, L. Yang, Multi-objective dynamic aperture optimization for storage rings, International Journal of Modern Physics A 31 (33) (2016) 1644019.

[28] Y. Li, W. Cheng, L. H. Yu, R. Rainer, Genetic algorithm enhanced by machine learning in dynamic aperture optimization, Physical Review Accelerators and Beams 21 (5) (2018) 054601.

[29] Y. Li, Y. Hao, K. Hwang, R. Rainer, A. He, A. Liu, Fast dynamic aperture optimization with forward-reversal integration, Nuclear Instruments and Methods in Physics Research Section A: Accelerators, Spectrometers, Detectors and Associated Equipment 988 (2021) 164936.

[30] A. J. Smola, B. Schölkopf, A tutorial on support vector regression, Statistics and computing 14 (3) (2004) 199-222.

[31] Y. Bengio, Y. Grandvalet, No unbiased estimator of the variance of k-fold cross-validation, Journal of machine learning research 5 (Sep) (2004) 10891105. 